\title{
Characterization of the malaria parasite protein PfTip, a novel invasion-related protein
}

\author{
XUEWU LIU*, YUXIAO HUANG ${ }^{*}$, JIAO LIANG, JUN WANG, YAN SHEN, YINGHUI LI and YAZHAO \\ Department of Pathogenic Biology, The Fourth Military Medical University, Xi'an, Shaanxi 710032, P.R. China
}

Received April 3, 2015; Accepted January 26, 2016

DOI: $10.3892 / \mathrm{mmr} .2016 .4910$

\begin{abstract}
Malaria is one of the most common infective diseases in the world. Invasion of host erythrocytes by the malaria parasite is crucial for pathogen survival and pathogenesis. Various proteins mediate parasite invasion and identification of novel invasion-related proteins may aid in elucidating the underlying molecular mechanism and new intervention strategies for malaria control. This study characterized the PfTip protein, a homolog of the human T-cell immunomodulatory protein, and examined its function in preventing parasite infection. Bioinformatics analysis and experimental validation were adopted in the present study. Bioinformatics analysis showed that PfTip has a $\beta$-propeller fold in its structure and is highly expressed at the early ring stage. TNFRSF14 was predicted to be a candidate interactant of PfTip. Further analyses showed that PfTip blockage by sera inhibited erythrocyte invasion by the malaria parasite. The protective effect of PfTip was further confirmed through in vivo analysis. To the best of our knowledge, this study is the first to provide evidence on the function of PfTip in erythrocyte parasite invasion. Additional assays involving the receptor of this protein are currently underway.
\end{abstract}

\section{Introduction}

Malaria is a tropical disease that results in millions of fatalities every year. The World Health Organization estimated that there were 207 million malaria cases and 627,000 estimated malaria-related fatalities in 2012 in their Malaria Report 2013 (1). Although the incidence and associated mortality of malaria are decreasing, the absolute number of malaria cases

Correspondence to: Professor Ya Zhao or Ms. Yinghui Li, Department of Pathogenic Biology, The Fourth Military Medical University, 17 Changlexi Street, Xi'an, Shaanxi 710032, P.R. China

E-mail: zhaoya@fmmu.edu.cn

E-mail: yinghlzb@fmmu.edu.cn

${ }^{*}$ Contributed equally

Key words: malaria parasite, PfTip, invasion, VCBS domain, structure remains significantly large due to the absence of an effective vaccine and the increase in drug-resistant parasite strains (2).

Erythrocyte invasion is the first step in the intraerythrocytic developmental cycle of Plasmodium falciparum. This parasite invades red blood cells (RBCs) through micronemes and rhoptry protein secretion, tight junction formation, and actomyosin assembly (3). A previous study established that disrupting the interactions between parasite and erythrocyte proteins prevents malaria parasite infection (4). Therefore, the identification of novel parasite proteins that are involved in RBC invasion has become a hotspot in malaria research. The inhibitions of several parasite proteins by gene knockout or antibodies has been demonstrated to provide protection against malaria infection. For instance, rhoptry neck protein 2 (PfRON2) can be secreted into RBCs and form complexes under the RBC membrane. However, several regions of PfRON2 can be left exposed to the extracellular environment. The microneme protein PfAMA1 bound to the exposed region of PfRON2 can form tight junctions between malaria parasite and RBCs. PfAMA1 knockout inhibits tight junction formation, thereby impairing erythrocyte invasion (5). In addition, the erythrocyte binding antigen PfEBA-175 is secreted onto the apical merozoite surface of malaria parasite. The binding of PfEBA175 to its RBC receptor gly-A triggers the secretion of rhoptry proteins. Gly-A cannot trigger rhoptry release when EBA-175 knockout mice are used (6). Other parasite proteins, such as PfRh5 and PfMtrap, also participate in the invasion process (7-9). The identification of novel proteins involved in the parasite invasion of RBCs is underway. Parasite proteins that are highly expressed at the schizont stage or early ring stage, or those that contain domains involved in cell-cell adhesion, are usually considered to be candidate invasion-related proteins.

The malaria parasite protein PfTip (Gene ID, PF3D7_0529000) is a homolog of the human ITFG1, which is a T-cell immunomodulatory protein (10). The protein contains four successive VCBS domains, which exist in multiple copies in long proteins from Vibrio, Colwellia, Bradyrhizobium, and Shewanella (hence the name VCBS), and fewer copies in proteins from bacteria. This domain is involved in cell-cell adhesion $(11,12)$. Proteins possessing this domain are usually involved in cell-cell contact. Whether or not PfTip can mediate parasite-erythrocyte interaction has yet to be determined. Determining the role of PfTip in RBC invasion by the malaria parasite may aid in elucidating the mechanism of invasion and provide a novel intervention strategy for malaria control. 
The present study aimed to characterize the PfTip protein. The sequence of PfTip was analyzed, its 3D structure was modeled, its expression feature was identified, and its interactant was predicted. The protective effect of PfTip inhibition was also evaluated through in vitro and in vivo experiments.

\section{Materials and methods}

Data sources and analysis tools. The protein sequences of PfTip and its homologs were obtained from PlasmoDB (http://plasmodb.org/plasmo/). Domain identification was performed using the PFAMdatabase (http://pfam.xfam.org/) (13). Protein-protein interactions were obtained from the BioGrid database (http://thebiogrid.org/) (14). Signal peptide and transmembrane domain were predicted using SignaIP and TMHMM servers (http://www.cbs.dtu.dk/services/), respectively. The secondary structure of PfTip was predicted using PSIPRED (http://bioinf.cs.ucl.ac.uk/psipred/) (15).

Structure modeling of PfTip. Building the structure of PfTip involves template identification, alignment of the template with the target, building of the model, and evaluation of the obtained structure (16). The PDB database was searched through HHsearch (HHsuite 3.0; Max Planck Gesellschaft, Berlin, Germany). Three templates with the highest sequence similarities were selected as templates to build PfTip models. Building the PfTip model included four steps: Template selection, sequence alignment, model building and evaluation of the obtained 3D structure. Initially, the PDB database was searched using HHsearch/HHpred to identify the templates. Three templates with the highest sequence similarities to the PfTip extracellular region were selected, including 2c4d.1.A, 4tqj.1.A and 4igl.1.A. Secondly, models were built based on selected templates using Swiss-model workspace. Finally, the local quality of built models was accessed by QMEAN6. The model built using 2c4d.1.A had the highest score and was considered to be the best structure. The alignment was then manually adjusted to improve the model quality further. The global qualities of the built models were verified using QMEAN6 (17). The model with the highest QMEAN6 Z-score was considered as the best 3D structure of PfTip. The local quality and structure of the best model were accessed through ProQ2 and PROCHECK $(18,19)$.

Fast fourier transform (FFT) analysis. FFT analysis was performed to detect genes specifically involved in the cell cycle (20). The procedure maps an expression signal in the time domain toward the frequency domain, thereby showing the amplitude of each frequency present in the expression signal. Through this method, it is possible to filter the expression signal that is inherently noisy or lacks differential expression and identify the major frequency of a particular gene in the life cycle of the malaria parasite. The formula of a simple FFT is expressed as follows:

$$
Y_{k}=\frac{1}{\sqrt{N}} \sum_{n=0}^{N-1} X_{n} e^{-j k\left(\frac{2 \pi}{N}\right) n}
$$

Where $\mathrm{N}$ is the length of expression signal and $k$ represents the frequency. The expression signal of PfTip was obtained from the published microarray data on the intraerythrocytic developmental cycle of $P$. falciparum (21). The major frequency of the PfTip expression signal can be determined through FFT analysis (22). The peak expression time point can be estimated using the following formula:

$$
T_{\text {peak }}=\left\{\begin{array}{c}
\frac{(-P)}{2 \pi} * 48 \text { if } P \text { is negative } \\
\frac{(-P)}{2 \pi} * 48+48 \text { if } P \text { is positive }
\end{array}\right.
$$

Where $P$ represents the phase of the major frequency. FFT analysis was implemented in MATLAB 2012b (MathWorks, Natick, MA, USA).

Expression and purification of the PfTIP-GST protein. The genomic DNA was isolated using the Genomic DNA Prep kit (Tiangen Biotech Co., Ltd., Beijing, China) and was set as the template for amplification. The DNA sequence encoding the ectodomain (48 to 674 aa) of PfTip was amplified through polymerase chain reaction (PCR) using the forward primer 5'-GCGGATCCAGGATA AAATCATTTTATGTTGAA GCA-3' and the reverse primer 5'-GCCTCGAGTTA TTT GGATGGATTAACTGAGAGTTG-3'. The components of the reaction system were: PrimerSTAR Max Premix (25 $\mu \mathrm{l})$, forward primer/reverse primer $(1 \mu \mathrm{l})$, template $(1 \mu \mathrm{l})$, water $(<50 \mu \mathrm{l})$. The thermal cycling conditions were as follows: $95^{\circ} \mathrm{C}$ for $5 \mathrm{~min}$, followed by 35 cycles of $94^{\circ} \mathrm{C}$ for $30 \mathrm{sec}, 52^{\circ} \mathrm{C}$ for $30 \mathrm{sec}, 72^{\circ} \mathrm{C}$ for $2 \mathrm{~min}$, and a final extension for $5 \mathrm{~min}$ at $72^{\circ} \mathrm{C}$. Following being digested with $\mathrm{BamHI}$ and $\mathrm{XhoI}$ (Takara Bio, Inc., Shiga, Japan), the fragment was inserted into pGEX-4T-1 (GE Healthcare, Fairfield, CT, USA) to express a GST fusion protein. Firstly, the vector pGEX-4T-1 and PfTip DNA fragment were digested by BamHI and XhoI for $2 \mathrm{~h}$ at $37^{\circ} \mathrm{C}$, followed by agarose gel electrophoresis. The target bands were cut and DNA fragments were harvested using a gel extraction kit (Omega Bio-Tek Inc., Norcross, GA, USA) and then the vector pGEX-4T-1 and PfTip DNA fragment were ligated overnight at $16^{\circ} \mathrm{C}$ using T4 ligase (New England Biolabs, Ipswich, MA, USA). The recombinant plasmids were transformed into XL-10 competent cells and were incubated on agar LB containing ampicillin for $12 \mathrm{~h}$. The transformed clones were picked and transferred into $10 \mathrm{ml}$ of LB. Following being incubated overnight at $37^{\circ} \mathrm{C}$, bacteria cells were harvested and plasmids were extracted. The correct open reading frame was confirmed by DNA sequencing. Following checking the correct sequence, the plasmids were transformed into Escherichia coli BL21 cells (Takara Bio., Inc.). First, the recombinant plasmids and competent cells were placed in tubes, mixed and then stored on ice for $30 \mathrm{~min}$. The tubes were then transferred into a preheated $42^{\circ} \mathrm{C}$ water bath for $90 \mathrm{sec}$. The tubes were immediately transferred to an ice bath and the cells were allowed to chill for at least $2 \mathrm{~min}$. Subsequently, $800 \mu \mathrm{l}$ of LB was added to the tube and the cells were agitated gently for $50 \mathrm{~min}$ in a rotary shaker. Finally, the transformed competent cells were transferred onto agar LB containing ampicillin and were incubated at $37^{\circ} \mathrm{C}$ for $12 \mathrm{~h}$. To express the recombinant protein PfTip-GST, IPTG (final concentration of $0.1 \mathrm{mM}$; Merck Millipore, Darmstadt, Germany) was added to the medium. Following further cultivation at $20^{\circ} \mathrm{C}$ for $10 \mathrm{~h}$, the 
bacterial samples were harvested. The parasite pellet was kept on ice and was sonicated for three cycles with $20 \mathrm{sec}$ pulses using an ultrasonicator (Ningbo Scientz Biotechnology Co., Ltd., Ningbo, China) with a $2 \mathrm{~mm}$ tip (60 watt model, $30 \%$ of maximum power). Sodium dodecyl sulfate-polyacrylamide gel electrophoresis was employed to determine PfTip expression. To purify the PfTIP-GST protein, the target band ( 97 kD) was cut, destained, ground with liquid nitrogen, and then dissolved in phosphate-buffered saline (PBS) containing $8 \mathrm{M}$ urea (Absin Bioscience, Shanghai, China). Following centrifugation at $12,000 \mathrm{x} \mathrm{g}$ for $10 \mathrm{~min}$, the supernatant was collected and the protein concentration was determined through a bicinchoninic acid (BCA) assay (Thermo Fisher Scientific, Inc., Waltham, MA, USA).

Generation of anti-PfTip sera. Two New Zealand white rabbits of the same breed were purchased from the Animal Care Facility at the Fourth Military Medical University (Xi'an, China). The present study was evaluated and approved by The Fourth Military Medical University Animal Care Committee. The rabbits were raised in standardized pathogen-free conditions at $20 \pm 2^{\circ} \mathrm{C}$ and $60 \pm 5 \%$ humidity, under a $12 / 12 \mathrm{~h}$ dark/light cycle. They were kept individually in steel cages with free access to food and tap water. To harvest the antisera, the rabbits were anesthetized by intramuscular injection of ketamine/xylazine (40 mg/kg; Sigma-Aldrich) and then blood was obtained through the carotid artery. To generate anti-PfTip sera, the rabbits were immunized by subcutaneously injecting $100 \mu \mathrm{g}$ of purified PfTip-GST protein mixed with complete Freund's adjuvant (Sigma-Aldrich, St. Louis, MO, USA). Animals injected with the adjuvant alone served as controls. Immunization was performed three times every 2 weeks. Sera were extracted 2 weeks after each immunization. The PfTip antibody titer of the sera was determined through enzyme-linked immunosorbent assay (ELISA). When the antibody titer was ideal, the rabbits were sacrificed and sera were extracted.

ELISA. ELISA was performed to determine the PfTip antibody titer of the sera. Each well was coated with $2 \mu \mathrm{g}$ purified PfTIP-GST protein at $37^{\circ} \mathrm{C}$ for $1 \mathrm{~h}$ and then at $4^{\circ} \mathrm{C}$ overnight. The following day, the wells were washed three times with PBS with Tween-20 (PBST) and then blocked at $37^{\circ} \mathrm{C}$ for $1 \mathrm{~h}$ with $5 \%$ non-fat milk. Sera samples diluted with blocking buffer (1:50; Beyotime Institute of Biotechnology, Shanghai, China) were added to each well and then incubated at $37^{\circ} \mathrm{C}$ for $1 \mathrm{~h}$. After washing with PBST, $100 \mu$ l diluted horseradish peroxidase (HRP)-conjugated polyclonal sheep anti-rabbit IgG (cat. no. A0208; Beyotime Institute of Biotechnology; 1:5,000) or polyclonal anti-mouse IgG (cat. no. A0216; Beyotime Institute of Biotechnology; 1:5,000) was added into each well and then incubated at $37^{\circ} \mathrm{C}$ for $1 \mathrm{~h}$. Excess IgG-HRP was removed by inverting the plate. TMB reagent (Beyotime Institute of Biotechnology) was added to each well to produce color. The absorbance of each well at $450 \mathrm{~nm}$ was measured and analyzed using a BIOBASE-EL10A ELISA microplate reader (Biobase Biodustry, Co., Ltd., Shandong, China).

Western blot analysis. Infected RBCs were lysed with $0.01 \%$ saponin (Sangon Biotech Co., Ltd., Shanghai, China) and then centrifuged at $12,000 \mathrm{x}$ g for $10 \mathrm{~min}$. The parasite pellet was suspended in PBS, lysed through four freeze/thaw cycles, and then sonicated for $3 \mathrm{X} 20 \mathrm{sec}$ on ice. The cell lysate was centrifuged at $12,000 \mathrm{x}$ g for $20 \mathrm{~min}$. The supernatant was collected, and the protein concentration was determined through a BCA assay. The cell lysate $(50 \mu \mathrm{g})$ was loaded per lane for separation through electrophoresis. The proteins were transferred to polyvinylidene difluoride (PVDF) membranes (EMD Millipore, Billerica, MA. USA), which were then blocked with 5\% non-fat milk in Tris-buffered saline for $1 \mathrm{~h}$ at room temperature. The anti-PfTip sera were added to the membranes and then incubated at $4^{\circ} \mathrm{C}$ overnight. After washing three times with PBST, the membranes were hybridized with polyclonal rabbit anti-human (1:1000; cat. no. T528; Signalway Antibody LLC, College Park, MD, USA) GST-Tag secondary antibody conjugated with IRDye 800 (LI-COR Biosciences, Lincoln, NE, USA) and then incubated for $1 \mathrm{~h}$. Blots were imaged using an infrared imaging system (Odyssey ${ }^{\circledR}$; LI-COR Biosciences).

Reverse transcription-quantitative PCR. Total RNA was extracted from the malaria parasite using TRIzol (Takara Bio, Inc.) and then reverse transcribed according to the manufacturer's instructions (Takara Bio Inc., Shiga, Japan). The obtained cDNA was used as the template for quantitative PCR. A PrimeScript ${ }^{\mathrm{TM}}$ RT reagent kit (Takara Bio, Inc.) was used for cDNA synthesis. The reaction system consisted of the following components: 5X PrimeScript Buffer $(2 \mu 1)$, $1 \mathrm{X}$ PrimeScript RT Enzyme mix I $(0.5 \mu \mathrm{l}), 50 \mu \mathrm{M}$ Oligo dT Primer $(0.5 \mu \mathrm{l}), 100 \mu \mathrm{M}$ random 6 mers $(0.5 \mu \mathrm{l})$, total RNA $(0.5 \mu \mathrm{g})$ and $\mathrm{RNase}$ Free $\mathrm{dH}_{2} \mathrm{O}$ (up to $10 \mu \mathrm{l}$ ). The primers (Sangon Biotech Co., Ltd.) used for the amplification of PfTip and the internal control gene (PF07_0073, Seryl-tRNA synthetase) were as follows: PfTip, 5'-GGGGTAATGCTC ATGGACCT-3' (forward) and 5'-GGGAAAT GTGCTGACT GGCT-3' (reverse); and PF07_0073, 5'-CAAGTAGCA GGT CATCGT GGT-3' (forward) and 5'-CAAGTTCGGCACATT CTTCCA-3' (reverse). The PCR reaction system consisted of $12.5 \mu \mathrm{l}$ of SYBR Green I Master mix (Takara Bio, Inc.), $500 \mathrm{nM}$ each primer, and $2 \mu 1$ of the template in a total volume of $25 \mu \mathrm{l}$. An Agilent Mx3000P QPCR System was used (Agilent Technologies, Inc., Santa Clara, CA, USA) for PCR. The thermal cycling conditions were as follows: $95^{\circ} \mathrm{C}$ for $5 \mathrm{~min}$, followed by 40 cycles of $94^{\circ} \mathrm{C}$ for $10 \mathrm{sec}$, and $60^{\circ} \mathrm{C}$ for $15 \mathrm{sec}$. The relative mRNA level of PfTip was quantified using the ${ }^{\Delta \Delta \mathrm{Cq}}$ method (23).

In vitro invasion assay. The invasion assay was conducted according a previous method (24). P. falciparum was cultured in RPMI-1640 medium supplemented with $10 \%$ human serum and erythrocytes ( $2 \%$ hematocrit) at $37^{\circ} \mathrm{C}$ and then flushed with a gas mixture containing $5 \% \mathrm{O}_{2}, 5 \% \mathrm{CO}_{2}$ and $90 \% \mathrm{~N}_{2}$. The medium was changed every $48 \mathrm{~h}$. For the invasion assay, $P$. falciparum was synchronized as previously described (25). When the parasite reached the trophozoite or schizont stage, MACS (Miltenyi Biotec GmbH, Bergisch Gladbach, Germany) magnetic bead separation column was used for separation of parasitized RBCs. Then the parasitemia was adjusted to 5\% and was cultured in 96-well plates with $180 \mu \mathrm{l}$ of medium. Each well contained $20 \mu \mathrm{l}$ of PfTip-immunized sera or control 

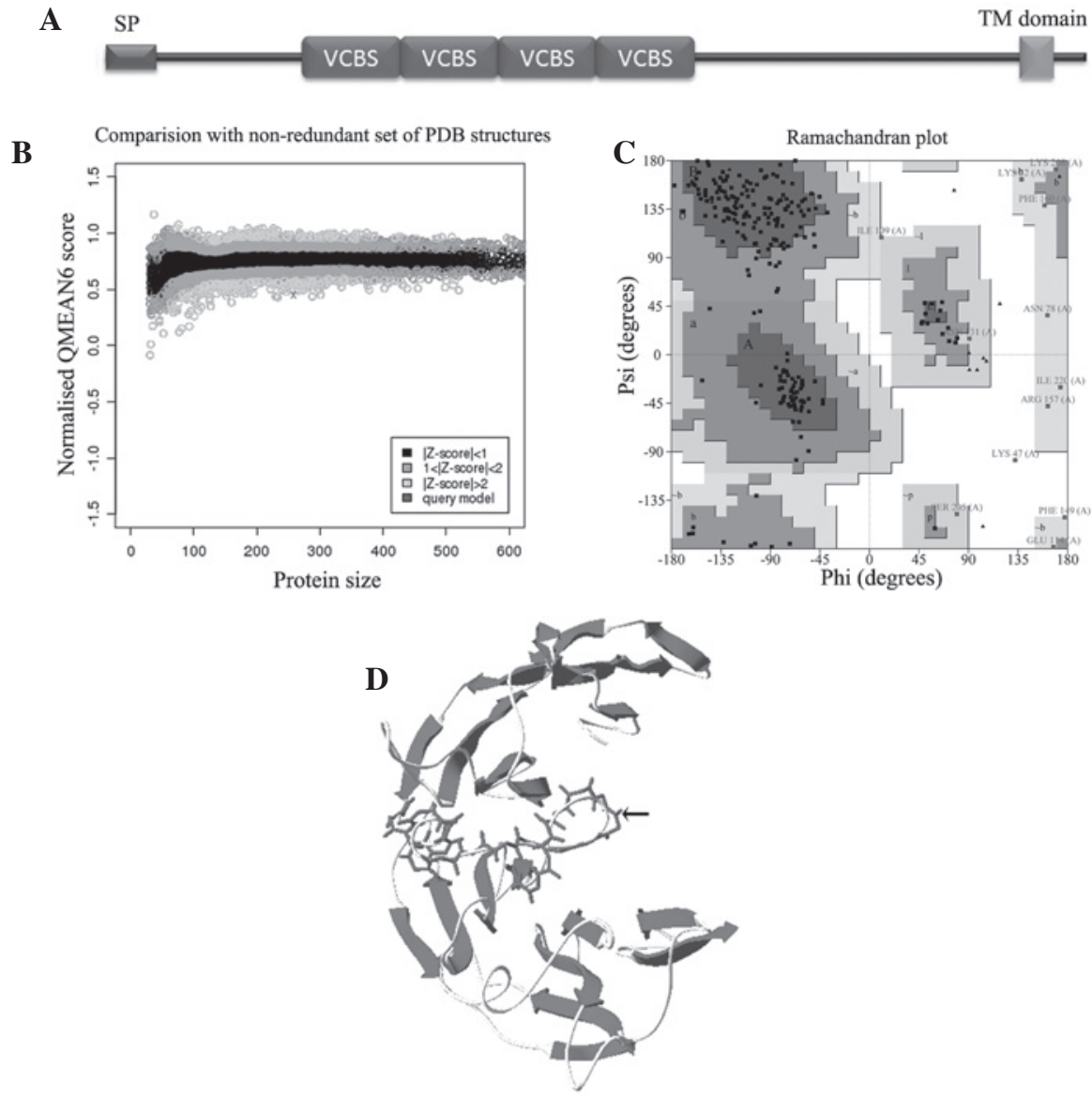

Figure 1. Sequential analysis and structural modeling of PfTip protein. (A) Domains and motifs identified in the PfTip protein. (B) Evaluation of the global quality of the PfTip model. Comparison of the normalized QMEAN6 score of PfTip with those of the non-redundant set of protein structure. Good models are generally located in the dark region. Moderate models are located in the gray region. Cross marker indicates a relatively moderate model according to its position near or in the gray region. (C) Ramachandran plot of PfTip obtained through modeling tool. (D) 3D model of PfTip consisting of four successive VCBS domains. Small arrow indicates that the loop was not well built. SP, signal peptide; TM, transmembrane domain.

Table I. Ramachandran plot statistics of the built 3D model of PfTip.

\begin{tabular}{lcc}
\hline Ramachandran plot statistics & No. of residues & (\%) \\
\hline $\begin{array}{l}\text { Residues in most favoured } \\
\text { regions [A,B,L] }\end{array}$ & 178 & 75.4 \\
$\begin{array}{l}\text { Residues in additional } \\
\text { allowed regions }\end{array}$ & 46 & 19.5 \\
$\begin{array}{l}\text { Residues in generously } \\
\text { allowed regions }\end{array}$ & 11 & 4.7 \\
$\begin{array}{l}\text { Residues in disallowed regions } \\
\text { Number of non-glycine and }\end{array}$ & 1 & 0.4 \\
$\begin{array}{l}\text { non-proline residues } \\
\text { Number of end-residues } \\
\text { (excl. Gly and Pro) }\end{array}$ & 236 & 100 \\
$\begin{array}{l}\text { Number of glycine residues } \\
\text { (shown as triangles) }\end{array}$ & 2 & \\
$\begin{array}{l}\text { Number of proline residues } \\
\text { Total number of residues }\end{array}$ & 7 & \\
\hline
\end{tabular}

sera. After $20 \mathrm{~h}$ of cultivation, Giemsa (Beijing Dingguo Biotechnology Co., Ltd., Beijing, China) staining smears were utilized to determine the ring stage parasitemia. An Olympus BX51 microscope (Olympus Corporation Tokyo, Japan) was used to observe Giemsa staining. The percent inhibition was calculated using the following formula:

$$
\frac{P_{\text {con }}-P_{p f}}{P_{\text {con }}} * 100
$$

Where $P_{p f}$ and $P_{c o n}$ represent the ring stage parasitemia after the addition of PfTip-GST sera and control sera, respectively.

In vivo protection test. A total of 14 female BALB/c mice aged 6-8 weeks were purchased from the Animal Care Facility at the Fourth Military Medical University and were divided into two groups, the treated group $(n=8)$ and the control group $(n=6)$. The animal experiments were approved by the Fourth Military Medical University Animal Care Committee. The mice were raised in pathogen-free conditions at $20 \pm 4^{\circ} \mathrm{C}$ and $60 \pm 5 \%$ humidity, under a 12/12 h dark/light cycle. The survival time of these mice were monitored for 25 days after intraperitoneal injection with Plasmodium berghei. All mice died within this period of time. The mice were subcutaneously injected with $10 \mu \mathrm{g}$ PfTIP-GST protein mixed with the adjuvant three times every 2 weeks, and mice injected with the adjuvant alone served as controls. Sera were extracted 2 weeks after each 
A

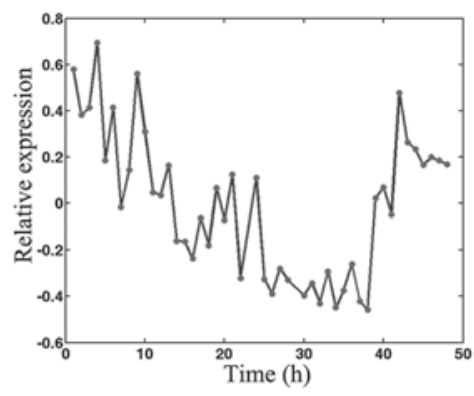

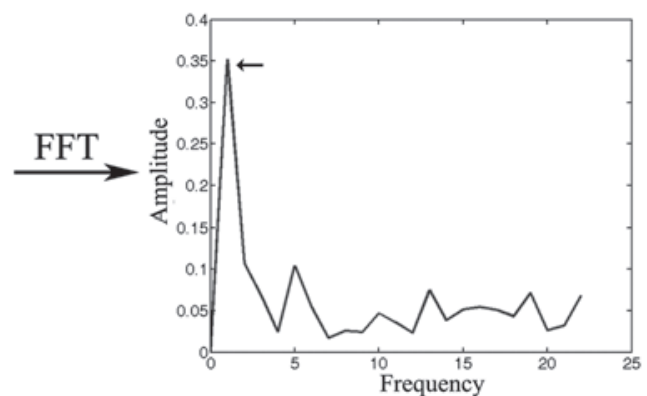

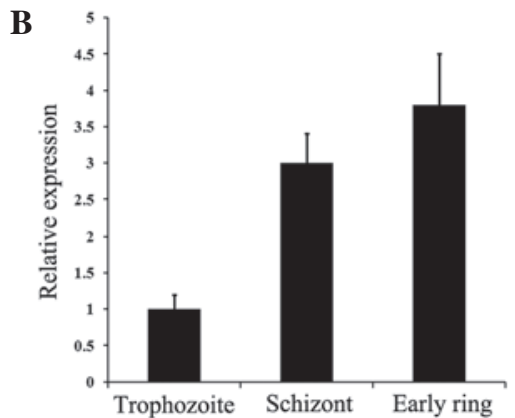

Figure 2. FFT analysis of PfTip expression and experimental validation. (A) FFT analysis of PfTip expression profile. Left panel, original mRNA level of PfTip detected in the intraerythrocytic developmental cycle of P.falciparum; Right panel, amplitude of each frequency identified through FFT analysis. Major frequency is the frequency with the largest amplitude (indicated by arrow). (B) Experimental validation of PfTip expression using reverse transcription-quantitative polymerase chain reaction. FFT, fast fourier transform.

injection. ELISA was performed to determine the antibody titer of the obtained sera. Mice with high levels of antibody titer were selected for further protection tests. The mice were administered with $1.0 \times 10^{6}$ Plasmodium berghei-infected RBCs through intraperitoneal injection. The survival time in days of the experimental mice was recorded. The animal experiments were permitted by the Fourth Military Medical University Animal Care Committee, and all animal studies were performed in compliance with the university institutional guidelines.

Statistical analysis. Statistical analysis was performed using statistic toolbox of MATLAB 2012b. Data are presented as the mean \pm standard deviation. One-way analysis of variance was used to compare two or more groups. The survival ratio was compared using Log-Rank test. $\mathrm{P}<0.05$ was considered to indicate a statistically significant difference.

\section{Results}

Sequential analysis and structural modeling of PfTip protein. PfTip, consisting of 719 aa, was encoded by a single exon in the P. falciparum genome. The protein sequence scans of PfTip by TMHMM and SignaIP indicated a signal peptide in its $\mathrm{N}$-terminus ( 1 to $28 \mathrm{aa}$ ) and a transmembrane domain in its C-terminus (678 to 697 aa) (Fig. 1A). Thus, PfTip was considered as a membrane protein and was expected to be expressed on the surface of the malaria parasite. However, this protein may not function as a receptor signal transducer from the outer to the inner parts of the cell as only 22 amino acids were predicted to be located in the cytoplasm. Searching the Pfam database for the ectodomain of this protein sequence, four successive VCBS domains (95 to 351 aa) were identified in this protein (Fig. 1A). The results of homolog identification through sequence alignment indicated that proteins containing the VCBS domain can be found in viruses, bacteria, protista, fungi, and animals but not in certain plants, including Oryza sativa and Arabidopsis thaliana. The results of secondary structure prediction by PSIPRED suggested the presence of $40 \beta$-strands in the PfTip ectodomain. However, no $\alpha$-helix was predicted in this region, implying that the structure of PfTip ectodomain is a member of the $\beta$-fold superfamily.
Table II. Prediction of PfTip interactant.

\begin{tabular}{lcc}
\hline Interactants $^{\mathrm{a}}$ & Signal peptide $^{\mathrm{b}}$ & TM domain $^{\mathrm{c}}$ \\
\hline CDC73 & $\mathrm{N}$ & $\mathrm{Y}$ \\
FAM118B & $\mathrm{N}$ & $\mathrm{N}$ \\
FBXO6 & $\mathrm{N}$ & $\mathrm{N}$ \\
HMOX2 & $\mathrm{N}$ & $\mathrm{Y}$ \\
MRPL44 & $\mathrm{N}$ & $\mathrm{N}$ \\
NUDT3 & $\mathrm{N}$ & $\mathrm{N}$ \\
SERBP1 & $\mathrm{N}$ & $\mathrm{N}$ \\
TAF1D & $\mathrm{N}$ & $\mathrm{N}$ \\
TNFRSF14 & $\mathrm{Y}$ & $\mathrm{Y}$ \\
UBC & $\mathrm{N}$ & $\mathrm{N}$ \\
\hline
\end{tabular}

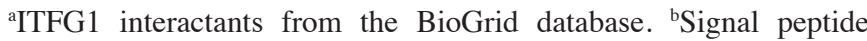
identified by SignaIP 4.1 . 'Transmembrane domain predicted using TMHMM 2.0. Y, found. N, not found.

To investigate the potential 3D structure of this protein, the PfTip extracellular region (95 to 356 aa) consisting of four successive VCBS domains was modeled using the Swiss-Model Workspace. The model built using the template Psathyrella velutina Lectin (PDB: 2c4d) had the highest QMEAN6 score ( 0.45 , normalized Z-score) and was selected as the 3D structure of PfTip (Fig. 1B, upper panel). The Ramachandran plot showed that $99.6 \%$ residues were placed in allowed regions, except for one residue (LYS47, Table I, and Fig. 1C). The built 3D structure (Fig. 1D) shows the presence of $\beta$-sheets, coils, loops, and turns but not $\alpha$-helixes. This finding is concurrent with the secondary structure prediction results. Furthermore, the $\beta$-strands in PfTip formed a $\beta$-propeller fold, which is also found in neuraminidase from the influenza virus (26). Proteins consisting of this fold usually participate in cell-cell contact and in pathogenic organism invasion of host cells (27), suggesting that PfTip may be an invasion-related protein. PfTip ectodomain has a low sequence similarity to the template (only $\sim 17.43 \%$ identity); thus, accurately modeling the native structure of PfTip is difficult. The assessment of the local quality of the obtained model revealed that the loop (99-122 aa) connecting two $\beta$-meanders was not 
A
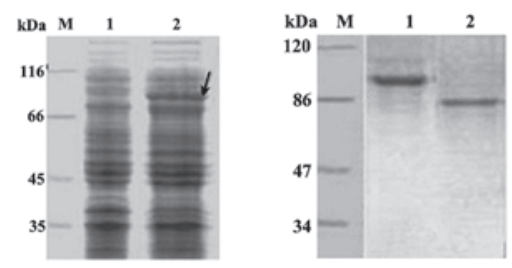

C

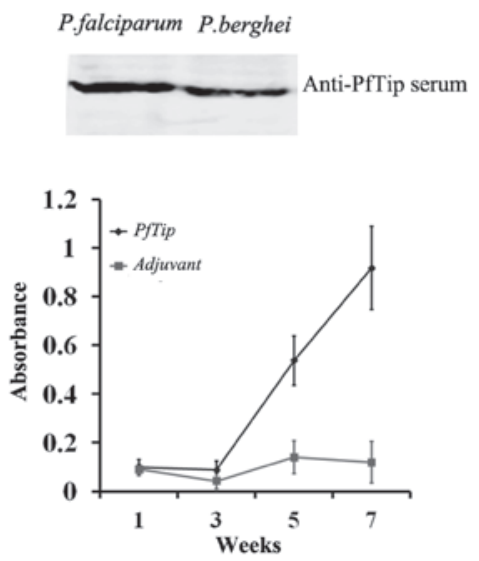

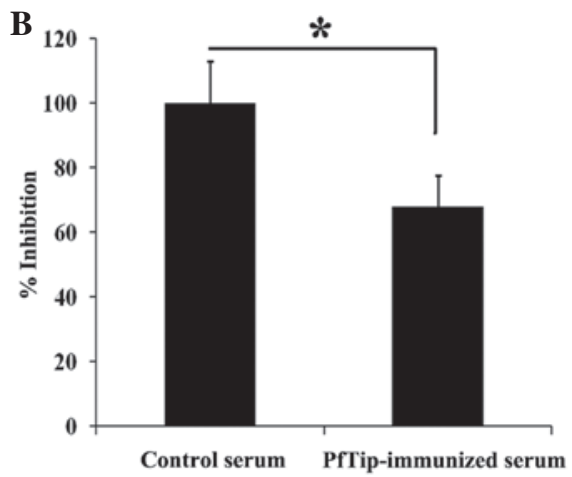

D

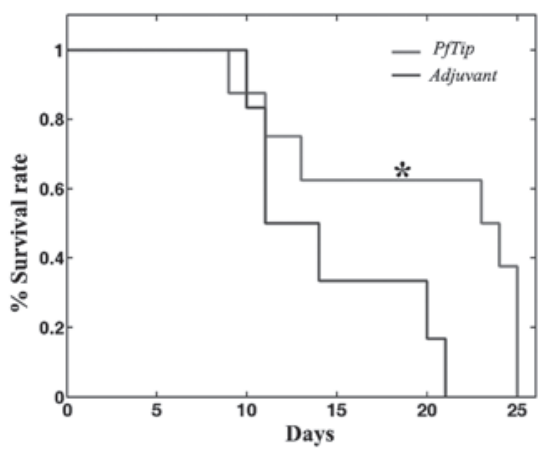

Figure 3. Blockage of PfTip protein inhibits parasite invasion of host cells and provides protection activity. (A) Preparation of PfTip-immunized serum. Left panel, sodium dodecyl sulfate-polyacrylamide gel electrophoresis of PfTip expression in E. coli BL21 cells. Arrow indicates PfTip expression. M, marker; Lane 1, without IPTG; Lane 2, $0.1 \mathrm{mM}$ IPTG. Right panel, sera specificity examination using western blot analysis. Lane 1, lysate of E. coli BL21 cells overexpressing PfTip; lane 2, P. falciparum lysate. (B) Suppression of parasite invasion ability using PfTip-immunized serum. One-way analysis of variance was used, ${ }^{*} \mathrm{P}<0.05$. (C) Determination of antibody specificity and titer using western blot analysis and ELISA. Upper panel, PfTip-immunized serum recognize PfTip and PbTip antigen; Lower panel, determination of antibody titer using ELISA. (D) Comparison of survival rates between two groups of experimental mice (PbTip group, $\mathrm{n}=8$; adjuvant group, $\mathrm{n}=6$ ). Log-rank test was used, $\mathrm{P}<0.05$. ELISA, enzyme-linked immunosorbent assay.

well built (Fig. 1D, indicated by small arrow). However, our model provided a clue for unraveling the function of PfTip.

Expression profile and interactant prediction of PfTip. Investigating the expression profile of PfTip may aid in elucidating the function of this protein. Proteins associated with invasion should be highly expressed on the surface of merozoites or in the schizont stage. Although gene expression is highly dynamic in the intraerythrocytic developmental cycle of $P$. falciparum, a striking nonstochastic periodicity can be observed for the majority of expression profiles. Considering the cyclical nature of gene expression, FFT analysis was performed to evaluate PfTip expression and its peak expression time point. After the parasite invasion of RBCs, PfTip expression decreased and had the lowest value in the trophozoite stage (Fig. 2A, left panel). However, PfTip expression began to rebound when the parasite reached the schizont stage. Compared with several parasite genes with more than one frequency in the asexual intraerythrocytic developmental cycle of P.falciparum, PfTip has only one frequency (Fig. 2A, right panel). Its peak expression time point was estimated to be $3.1 \mathrm{~h}$ ( $T_{\text {peak }}$ calculation in methods), suggesting that PfTip was highly expressed at the early ring stage. Further experimental validation of PfTip expression by RT-qPCR indicated that this protein was highly expressed at the schizont and early ring stages. The expression level of PfTip at the early ring stage was almost three times higher than that at the trophozoite stage. Thus, based on its expression pattern, PfTip is hypothesized to be an invasion-related protein.

The prediction of proteins interacting with PfTip may aid in elucidating the mechanism by which PfTip mediates $\mathrm{RBC}$ invasion by the malaria parasite. Considering that the protein domain is evolutionarily conserved and that protein-protein interactions are predominantly achieved through domain-domain interactions, a homolog method was employed to predict the interactant of PfTip. In this method, the homologs of a protein are considered to have the propensity to bind with the interactants of the protein. Basing on this concept, the BioGrid database was searched and 10 proteins that reportedly physically interact with ITFG1, the homolog of PfTip in human were identified. However, no protein was found to interact with the PfTip homologs from other species, including Caenorhabditis elegans, Drosophila melanogaster, and Mus musculus. The interaction partner of PfTip, as a membrane protein, should be expressed on the surface of erythrocytes. Combining the prediction results of SignaIP and TMHMM, nine proteins were removed that were not expressed on the surface of RBCs, leaving TNFRSF14 as the candidate partner of PfTip (Table II). TNFRSF14 has previously been demonstrated to be a receptor of BTLA (28). A previous study showed that TNFRSF14 functions as a co-stimulator of $\mathrm{T}$ cells (29). Whether or not TNFRSF14 
physically interacts with PfTip requires further experimental validation.

Experimental validation of PfTip function in invasion. Considering that PfTip contains the VCBS domain in its structure and is highly expressed in the early ring and schizont stages, it was hypothesized that PfTip is an invasion-related protein. Thus, PfTip blockage may inhibit malaria parasite invasion and confer protection. To experimentally validate the function of PfTip in erythrocyte invasion, a plasmid expressing the fusion protein PfTip-GST (Fig. 3A, left panel) was constructed. Then, rabbits were immunized three times using the purified PfTip-GST protein to generate an antibody recognizing the PfTip protein. The results of western blot analysis indicated that the obtained rabbit serum can specifically recognize PfTip (Fig. 3A, right panel).

The interaction between PfTip and its receptor was blocked using the obtained immune serum to evaluate whether or not PfTip blockage inhibits parasite invasion. The ring stage parasitemia was examined and used for percent inhibition computation. As shown in Fig. 3B, the ring stage parasitemia observed in the PfTip-immunized group was significantly lower than that in the control group $(\mathrm{P}<0.05)$. The parasitemia decreased by almost $35 \%$. This finding demonstrated that PfTip blockage by the immune serum inhibited RBC invasion by the malaria parasite. However, the underlying molecular mechanism requires further investigation as the surface protein of RBCs that interacts with PfTip has not yet been identified.

To test the protection activity of PfTip, an in vivo experiment was performed. Given that the protective effect of PfTip cannot be validated in humans, a mouse model was used instead. Mice were immunized with the PfTip-GST protein three times to produce a relatively high level of antibodies in mouse sera (Fig. 3C, upper panel). The PfTip-immunized serum can also recognize PbTip due to the high sequence similarity $(\sim 85 \%)$ between PbTip and PfTip (Fig. 3C, lower panel). This result suggests that the antibody induced by PfTip can effectively block the interactions between PbTip and its partner. To confirm the protective effect of PbTip blockage, the mice intraperitoneally injected with $P$. berghei were monitored for up to 25 days. Despite the lack of difference in survival rate between the two groups, the survival curve was delayed when PfTip was injected (Fig. 3D, lower panel). The mean survival time of these two groups were 12.5 and 23.5 days, respectively. Statistical analysis indicates that the difference was significant $(\mathrm{P}=0.0496)$. This result demonstrates that PfTip injection into mice confer a slight resistance to the lethal malaria parasite infection.

\section{Discussion}

Erythrocyte invasion by malaria parasite is a complex biological process. Various parasite proteins, including PfAMA1 and PfRh5, participate in this invasion process $(30,31)$. The interactions between parasite and erythrocyte proteins facilitate the adhesion of the parasite to RBCs, subsequently initiating the invasion process. The identification of proteins associated with this process may aid in elucidating the molecular mechanism related to the parasite invasion of RBCs and potentially provide a novel strategy for malaria control. In the present study, PfTip was characterized and demonstrated that PfTip consists of four successive VCBS domains and PfTip expression at the schizont and early ring stage was relatively higher compared with that observed in the trophozoite stage. In addition, PfTip blockage by sera inhibits parasite invasion and provides protection. However, further studies regarding the protection mechanism employed by PfTip are required.

Regarding the structure of PfTip, the model of PfTip ectodomain was generated through homology modeling. Although the local quality of several regions of this model was not ideal due to the low sequence similarity with the template, the score of the global quality revealed that the developed model was relatively reliable. The structure of the ectodomain composed of four successive VCBS domains shows a part of the $\beta$-propeller fold. This fold is a type of a $\beta$-strand architecture characterized by several $\beta$-sheets toroidally arranged around a central axis (12). The assembly of multiple $\beta$-sheets repeatedly forms the structure domain responsible for protein-protein interaction. This fold can be found in several enzymes and membrane proteins $(26,32)$. For instance, the influenza virus protein neuraminidase possesses a $\beta$-propeller fold. This protein is present in the virus envelope and catalyzes the cleavage of sialic acid moieties from cell membrane proteins to aid in the invasion of host cells. Thus, the built model suggested that PfTip may be an invasion-related protein. The study on the expression pattern of PfTip also supported this hypothesis as PfTip is highly expressed at the early ring and schizont stages.

If PfTip is an invasion-related protein, then its blockage should theoretically inhibit RBC invasion by the malaria parasite. This speculation was supported by the results of in vivo and in vitro experiments. In the in vitro experiment, PfTip blockage using sera significantly suppressed the parasite invasion of RBCs and subsequently reduced ring parasitemia. In the in vivo experiment, the survival curve of the PfTip-immunized mice was delayed, indicating the protective effect of PfTip injection. The delay in the survival curve may be attributed to two reasons. First, the antibody induced through PfTip injection impaired the invasion ability of malaria parasite and thus reduced the parasite burden required to initiate the pathways of severe malaria. Second, PfTip probably enhanced host immunity against parasite infection. This phenomenon can be attributed to the fact that ITFG1, a homolog of PfTip in humans, can stimulate the $\mathrm{T}$ cell secretion of $\gamma$-interferon (10). The interactions between the T-cell receptor and the VCBS domain were evolutionarily conserved. Thus, the injection of PfTip in mice may also lead to $\mathrm{T}$ cell activation, subsequently enhancing the host immunity against parasite infection. Although the co-stimulator TNFRSF14 was predicted to be the receptor of PfTip protein, we did not experimentally validate this interaction as the expression of this protein in mammalian cells was not viable even under code optimization. The validation of this predicted interaction will be a focus of future studies.

In conclusion, the present study revealed that PfTip was a novel invasion-related protein. As inhibition of PfTip protein inhibited malaria parasite invasion and conferred protection, this protein may be considered a candidate vaccine. Identification of the PfTip-binding receptor on the surface of erythrocytes is required in order to understand how PfTip protein aids parasites invading RBCs. 


\section{Acknowledgements}

This study was supported by the Nature Science Foundation of China (grant no. 81572013) and the China Postdoctoral Science Foundation (grant no. 2015M582796). The authors would like to thank Professor Ya Zhao for critically revising the manuscript.

\section{References}

1. World health organization: World malaria report 2013 http://www.who.int/malaria/publications/world_malaria_ report_2013/en/2013.

2. Ashley EA, Dhorda M, Fairhurst RM, Amaratunga C, Lim P, Suon S, Sreng S, Anderson JM, Mao S, Sam B, et al: Spread of artemisinin resistance in Plasmodium falciparum malaria. N Engl J Med 371: 411-423, 2014.

3. Sharma P and Chitnis CE: Key molecular events during host cell invasion by Apicomplexan pathogens. Curr Opin Microbiol 16: 432-437, 2013

4. Patarroyo ME, Bermúdez A and Patarroyo MA: Structural and immunological principles leading to chemically synthesized, multiantigenic, multistage, minimal subunit-based vaccine development. Chem Rev 111: 3459-3507, 2011.

5. Giovannini D, Späth S, Lacroix C, Perazzi A, Bargieri D, Lagal V, Lebugle C, Combe A, Thiberge S, Baldacci P, et al: Independent roles of apical membrane antigen 1 and rhoptry neck proteins during host cell invasion by apicomplexa. Cell Host Microbe 10: 591-602, 2011.

6. Singh S, Alam MM, Pal-Bhowmick I, Brzostowski JA and Chitnis CE: Distinct external signals trigger sequential release of apical organelles during erythrocyte invasion by malaria parasites. PLoS Pathog 6: e1000746, 2010.

7. Baum J, Chen L, Healer J, Lopaticki S, Boyle M, Triglia T, Ehlgen F, Ralph SA, Beeson JG and Cowman AF: Reticulocyte-binding protein homologue 5-an essential adhesin involved in invasion of human erythrocytes by plasmodium falciparum. Int J Parasitol 39: 371-380, 2009.

8. Crosnier C, Bustamante LY, Bartholdson SJ, Bei AK, Theron M, Uchikawa M, Mboup S, Ndir O, Kwiatkowski DP, Duraisingh MT, et al: Basigin is a receptor essential for erythrocyte invasion by plasmodium falciparum. Nature 480: 534-537, 2011.

9. Bartholdson SJ, Bustamante LY, Crosnier C, Johnson S, Lea S, Rayner JC and Wright GJ: Semaphorin-7A is an erythrocyte receptor for $P$. falciparum merozoite-specific TRAP homolog, MTRAP. PLoS Pathog 8: e1003031, 2012.

10. Fiscella M, Perry JW, Teng B, Bloom M, Zhang C, Leung K, Pukac L, Florence K, Concepcion A, Liu B, et al: TIP, a T-cell factor identified using high-throughput screening increases survival in a graft-versus-host disease model. Nat Biotechnol 21: 302-307, 2003.

11. Cioci G, Mitchell EP, Chazalet V, Debray H, Oscarson S, Lahmann M, Gautier C, Breton C, Perez S and Imberty A: Beta-propeller crystal structure of psathyrella velutina lectin: An integrin-like fungal protein interacting with monosaccharides and calcium. J Mol Biol 357: 1575-1591, 2006.

12. Kopec KO and Lupas AN: $\beta$-Propeller blades as ancestral peptides in protein evolution. PloS One 8: e77074, 2013.

13. Finn RD, Bateman A, Clements J, Coggill P, Eberhardt RY, Eddy SR, Heger A, Hetherington K, Holm L, Mistry J, et al: Pfam: The protein families database. Nucleic Acids Res 42 (Database Issue): D222-D230, 2014
14. Chatr-Aryamontri A, Breitkreutz BJ, Heinicke S, et al: The BioGRID interaction database: 2013 update. Nucleic Acids Res 41 (Database Issue): D816-D823, 2013.

15. Buchan DW, Minneci F, Nugent TC, Bryson K and Jones DT: Scalable web services for the PSIPRED protein analysis workbench. Nucleic Acids Res 41 (Web Server Issue): W349-W357, 2013

16. Bordoli L, Kiefer F, Arnold K, Benkert P, Battey J and Schwede T: Protein structure homology modeling using SWISS-MODEL workspace. Nat Protoc 4: 1-13, 2009.

17. Benkert P, Biasini $\mathrm{M}$ and Schwede T: Toward the estimation of the absolute quality of individual protein structure models. Bioinformatics 27: 343-350, 2011.

18. Laskowski RA, Moss DS and Thornton JM: Main-chain bond lengths and bond angles in protein structures. J Mol Biol 231: 1049-1067, 1993.

19. Ray A, Lindahl E and Wallner B: Improved model quality assessment using ProQ2. BMC Bioinformatics 13: 224, 2012.

20. Kim BR, Zhang L, Berg A, Fan J and Wu R: A computational approach to the functional clustering of periodic gene-expression profiles. Genetics 180: 821-834, 2008.

21. Bozdech Z, Llinás M, Pulliam BL, Wong ED, Zhu J and DeRisi JL: The transcriptome of the intraerythrocytic developmental cycle of plasmodium falciparum. PLoS Biol 1: E5, 2003.

22. Liu X, Huang Y, Liang J, Zhang S, Li Y, Wang J, Shen Y, Xu Z and Zhao Y: Computational prediction of protein interactions related to the invasion of erythrocytes by malarial parasites. BMC Bioinformatics 15: 393, 2014.

23. Livak KJ and Schmittgen TD: Analysis of relative gene expression data using real-time quantitative PCR and the 2(-Delta Delta C(T)) method. Methods 25: 402-408, 2001.

24. Recuenco FC, Kobayashi K, Ishiwa A, Enomoto-Rogers Y, Fundador NG, Sugi T, Takemae H, Iwanaga T, Murakoshi F, Gong $\mathrm{H}$, et al: Gellan sulfate inhibits plasmodium falciparum growth and invasion of red blood cells in vitro. Sci Rep 4: 4723, 2014.

25. Iwanaga T, Sugi T, Kobayashi K, Takemae H, Gong H, Ishiwa A, Murakoshi F, Recuenco FC, Horimoto T, Akashi H and Kato K: Characterization of plasmodium falciparum cdc2-related kinase and the effects of a CDK inhibitor on the parasites in erythrocytic schizogony. Parasitol Int 62: 423-430, 2013.

26. Smith BJ, Colman PM, Von Itzstein M, Danylec B and Varghese JN: Analysis of inhibitor binding in influenza virus neuraminidase. Protein Sci 10: 689-696, 2001.

27. Chao KL, Tsai IW, Chen C and Herzberg O: Crystal structure of the Sema-PSI extracellular domain of human RON receptor tyrosine kinase. PloS One 7: e41912, 2012.

28. Shui JW, Steinberg MW and Kronenberg M: Regulation of inflammation, autoimmunity and infection immunity by HVEM-BTLA signaling. J Leukoc Biol 89: 517-523, 2011.

29. Chen L and Flies DB: Molecular mechanisms of T cell co-stimulation and co-inhibition. Nat Rev immunol 13: 227-242, 2013.

30. Yap A, Azevedo MF, Gilson PR, Weiss GE, O'Neill MT, Wilson DW, Crabb BS and Cowman AF: Conditional expression of apical membrane antigen 1 in plasmodium falciparum shows it is required for erythrocyte invasion by merozoites. Cell Microbiol 16: 642-656, 2014.

31. Shen Y, Wang J, Liu X, Liang J, Huang Y, Liu Z, Zhao YA and Li Y: Blockade of plasmodium falciparum erythrocyte invasion: New assessment of anti-Plasmodium falciparum reticulocyte-binding protein homolog 5 antibodies. Exp Ther Med 9: 1357-1362, 2015.

32. Wu D, Hu Q, Yan Z, Chen W, Yan C, Huang X, Zhang J, Yang P, Deng H, Wang J, et al: Structural basis of ultraviolet-B perception by UVR8. Nature 484: 214-219, 2012. 